\title{
Strong silk fibers containing cellulose nanofibers generated by a bioinspired microfluidic chip
}

Li Lu, ${ }^{1}$ Suna Fan, ${ }^{1}$ Qianqian Niu, ${ }^{1}$ Qingfa Peng, ${ }^{1}$ Lihong Geng, ${ }^{2}$ Gesheng Yang, ${ }^{1}$ Huili Shao, ${ }^{1}$

Benjamin S. Hsiao, ${ }^{*}, 3$ and Yaopeng Zhang ${ }^{*}, 1$

1 State Key Laboratory for Modification of Chemical Fibers and Polymer Materials,

Shanghai Belt and Road Joint Laboratory of Advanced Fiber and Low-Dimension Materials,

College of Materials Science and Engineering, Donghua University, 2999 North Renmin

Road, Songjiang, Shanghai 201620, P.R. China

2 Key Laboratory of Polymer Materials and Products of Universities in Fujian, Fujian

University of Technology, 33 Xuefu South Road, New University District, Fujian 350118 ,

P.R. China

3 Department of Chemistry, Stony Brook University, Stony Brook, NY 11794, USA ${ }^{1}$

number of pages: 4

number of figures: 2

number of table: 1

* Corresponding authors, E-mail address: zyp@dhu.edu.cn(Y.Z);

benjamin.hsiao@stonybrook.edu (B.H) 

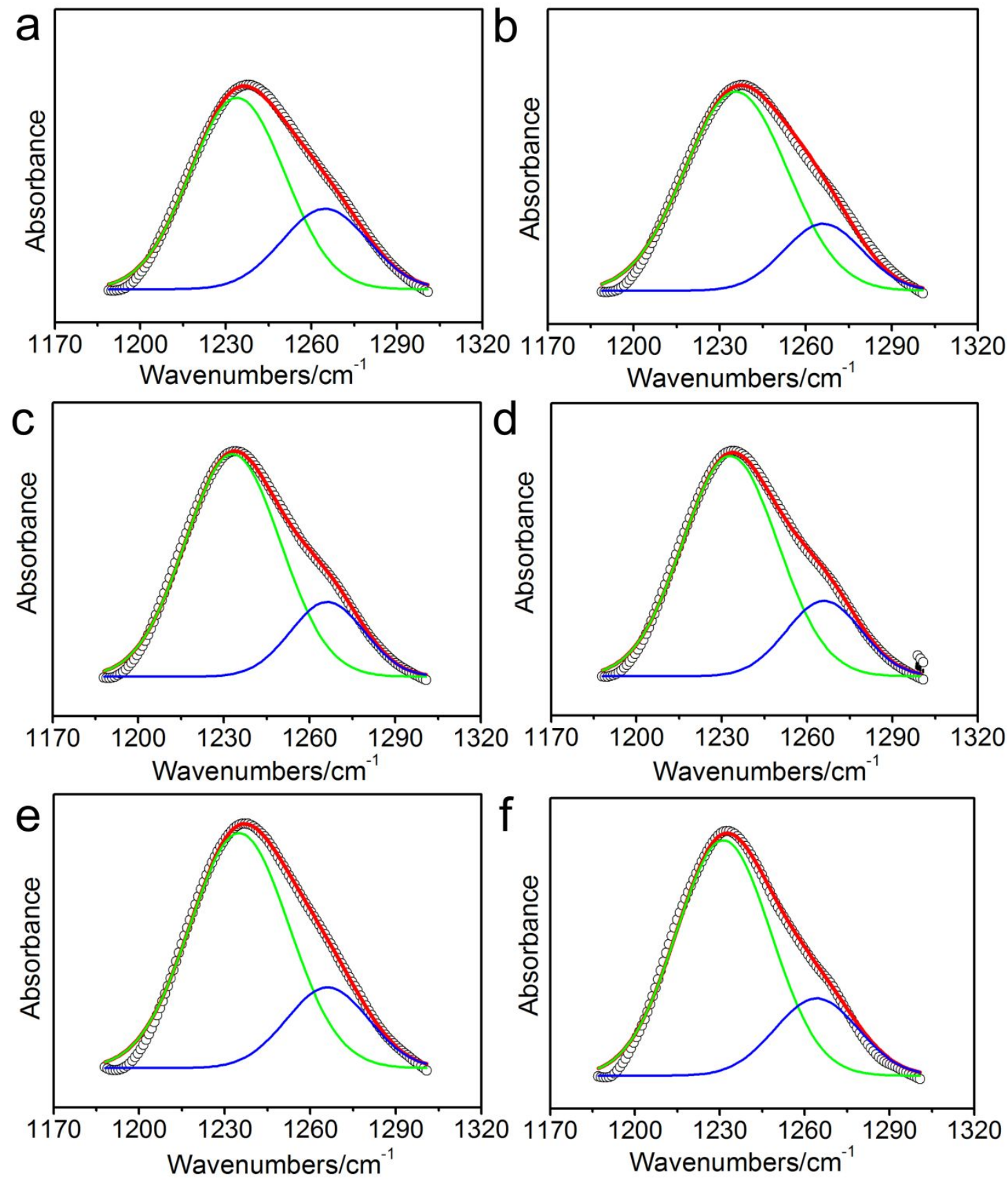

Figure S1. The deconvolution of FTIR in amide III band. (a) De-silk and RSF/CNF fibers:(b) RSF, (c) RSF/CNF -0.5, (d) RSF/ CNF -1, (e) RSF/ CNF -2, and (f) RSF/ CNF -3. 
Table S1. Mechanical properties of De-silk and RSF/CNF fibers.

\begin{tabular}{ccccccc}
\hline Samples & $\begin{array}{c}\text { Diameter } \\
(\mu \mathrm{m})\end{array}$ & $\begin{array}{c}\text { Stress } \\
(\mathrm{MPa})\end{array}$ & $\begin{array}{c}\text { Strain } \\
(\%)\end{array}$ & $\begin{array}{c}\text { Modulus } \\
(\mathrm{GPa})\end{array}$ & $\begin{array}{c}\text { Breaking energy } \\
(\mathrm{KJ} / \mathrm{Kg})\end{array}$ & $\begin{array}{c}\text { Birefringence } \\
\text { index }\end{array}$ \\
\hline De-silk & $10.8 \pm 1.9$ & $265.2 \pm 89.3$ & $12.7 \pm 4.0$ & $3.6 \pm 2.0$ & $16.2 \pm 9.5$ & $0.0471 \pm 0.0097$ \\
RSF & $8.4 \pm 1.1$ & $307.2 \pm 22.7$ & $17.4 \pm 4.9$ & $7.1 \pm 2.2$ & $29.5 \pm 8.9$ & $0.0375 \pm 0.0073$ \\
$\mathrm{RSF} / \mathrm{CNF}-0.5$ & $8.3 \pm 1.8$ & $374.8 \pm 40.1$ & $14.7 \pm 4.9$ & $7.3 \pm 2.3$ & $28.7 \pm 11.1$ & $0.0453 \pm 0.0082$ \\
$\mathrm{RSF} / \mathrm{CNF}-1$ & $7.9 \pm 2.0$ & $486.9 \pm 106.6$ & $15.8 \pm 4.7$ & $9.3 \pm 2.5$ & $41.0 \pm 18.1$ & $0.0471 \pm 0.0012$ \\
$\mathrm{RSF} / \mathrm{CNF}-2$ & $7.2 \pm 0.9$ & $356.9 \pm 47.4$ & $12.8 \pm 4.4$ & $7.9 \pm 2.5$ & $23.1 \pm 8.9$ & $0.0418 \pm 0.0095$ \\
$\mathrm{RSF} / \mathrm{CNF}-3$ & $7.2 \pm 1.5$ & $356.0 \pm 46.9$ & $15.2 \pm 3.1$ & $7.4 \pm 1.5$ & $26.3 \pm 6.9$ & $0.0431 \pm 0.0029$ \\
\hline
\end{tabular}



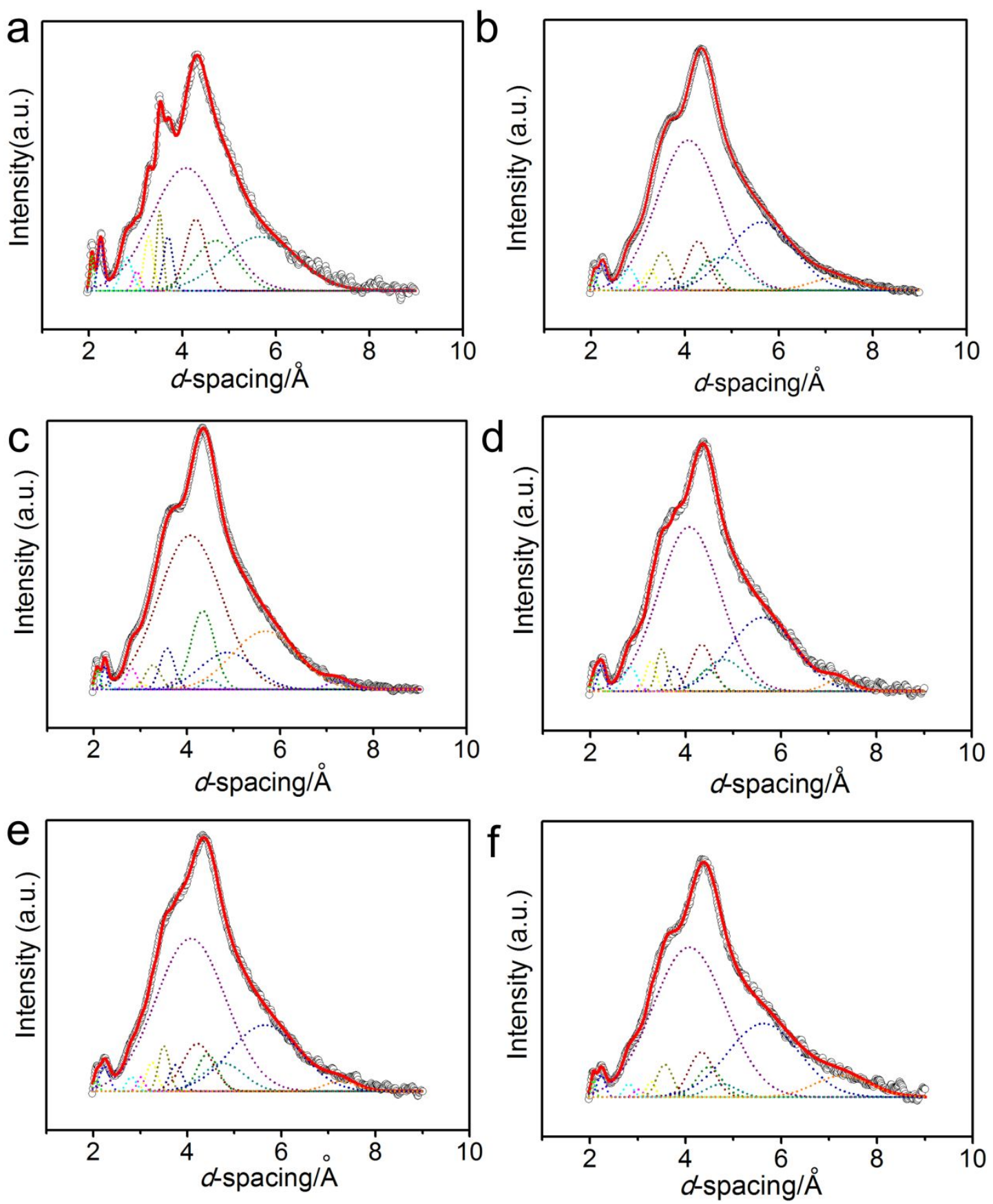

Figure S2. The deconvolution of 1 D SR-WAXD diffractograms of (a) De-silk and RSF/ CNF fibers:(b) RSF , (c) RSF/ CNF - 0.5, (d) RSF/ CNF - 1, (e) RSF/ CNF - 2, and (f) RSF/ CNF - 3. 